Artículo

\title{
Evaluación de métodos de recirculación de solución nutritiva para la producción de jitomate en ciclos cortos
}

Felipe Sánchez del Castillo ${ }^{1}$

Alan Cabañas Díaz ${ }^{1}$

Joel Pineda Pineda ${ }^{1}$

Lucila González Molina²

Esaú del Carmen Moreno Pérez ${ }^{1 \S}$

${ }^{1}$ Universidad Autónoma Chapingo. Carretera México-Texcoco km 38.5, Texcoco, Estado de México, México. CP. 56230. Tel. 595 9521500, ext. 6313. (fsanchezdelcastillo@yahoo.com.mx; alanmaestriaenciencias@gmail.com; pinedapjoel@yahoo.com.mx. ${ }^{2}$ Campo Experimental Valle de MéxicoINIFAP. Carretera Los Reyes-Texcoco km 13.5, Coatlinchán, Texcoco, Estado de México. CP. 56250. (gonzalez.lucila@inifap.gob.mx).

${ }^{\S}$ Autor para correspondencia: esaump10@yahoo.com.mx.

\section{Resumen}

El jitomate (Solanum lycopersicum L.) es la hortaliza más cultivada en invernadero e hidroponía. Por facilidad de manejo, los sistemas hidropónicos abiertos (sin recirculación de la solución nutritiva) con sustrato son los más utilizados en el mundo. Los sistemas cerrados (con recirculación), permiten ahorrar agua y fertilizantes, pero su manejo técnico es difícil, pues con el tiempo, se van acumulando los iones menos consumidos por la planta, que, al recircularlos, ocasionan desequilibrios nutrimentales e incrementos en la $\mathrm{CE}$ a niveles que afectan el crecimiento y rendimiento, además del alto riesgo de dispersarse enfermedades sobre todo con ciclos de cultivo tan largos como convencionalmente se maneja el jitomate. El objetivo fue comparar tres métodos de recirculación de solución nutritiva contra un sistema abierto, en el comportamiento agronómico de jitomate manejado en alta densidad de población con despunte para cosechar solamente tres racimos por planta. El diseño fue bloques completos al azar con cuatro tratamientos y siete repeticiones, con unidad experimental de $20 \mathrm{~m}^{2}$. Se evaluaron variables morfológicas, peso seco y rendimiento. Exceptuando altura y diámetro de tallo, ninguna variable mostró diferencia estadística entre tratamientos. Se concluye que, con el manejo de las plantas de jitomate despuntadas para cosechar tres racimos, en un ciclo tan corto como 110 días de trasplante a fin de cosecha, es factible utilizar cualquier sistema de recirculación de solución nutritiva sin desbalances nutricionales, de manera que, al comparar estos métodos con un sistema abierto, no se afecta el crecimiento ni el rendimiento de las plantas, ahorrándose agua y fertilizantes.

Palabras clave: invernadero, sistemas hidropónicos cerrados, solución nutritiva.

Recibido: enero de 2021

Aceptado: febrero de 2021 


\section{Introducción}

El jitomate (Solanum lycopersicum L.) es una de las hortalizas que más se manejan bajo condiciones de invernadero (SIAP, 2018). Debido a lo intensivo de su manejo en condiciones de invernadero se favorece el establecimiento progresivo de patógenos en el suelo después de algunos ciclos de cultivo (Takahashi, 1984). La compactación, la acumulación de sales, los desequilibrios nutrimentales y la proliferación de malezas son otros factores que afectan negativamente el rendimiento (Liang et al., 2006).

Una alternativa para resolver estos problemas ha sido la hidroponía o cultivo sin suelo, donde las plantas crecen en una solución nutritiva, con o sin un sustrato como medio de soporte (Urrestarazu, 2015), permitiendo desarrollar el sistema radical de las plantas en completa independencia del suelo. En condiciones de invernadero la hidroponía le va ganando terreno a la producción en suelo debido a que se logra mayor eficiencia y control del riego y la nutrición mineral, así como por la ausencia inicial de plagas, enfermedades y malezas y porque es más fácil la esterilización de los sustratos (Alarcón, 2006; Raviv y Lieth, 2008).

Con el invernadero se logra el control de los requerimientos de las plantas en cuanto a los factores climáticos, mientras que los sistemas hidropónicos se diseñan con el objetivo de poner a la raíz en las condiciones ambientales más adecuadas para su óptimo funcionamiento. La conjunción de ambas tecnologías representa la agricultura comercial más avanzada con la que se cuenta en la actualidad a nivel mundial (Sánchez y Moreno, 2017).

En los sistemas hidropónicos basados en el uso de sustratos inertes, para que las plantas de jitomate crezcan sin limitaciones nutricionales, la solución nutritiva debe tener un $\mathrm{pH}$ entre 5.5 a 6.5 , una conductividad eléctrica (CE) entre 1.5 y $3 \mathrm{dS} \mathrm{m}^{-1}$ y los nutrimentos minerales deben estar disociados en forma iónica en las proporciones y concentraciones adecuadas según sus tasas de absorción y en condiciones que eviten precipitados y antagonismos (Adams, 2004).

La planta modifica el consumo de nutrimentos en función de sus fases de crecimiento y desarrollo, condiciones climáticas, y características de la solución nutritiva como la $\mathrm{CE}, \mathrm{pH}$, temperatura y oxígeno disuelto (Sonneveld y Voogt, 2009; Velazco et al., 2012; Urrestarazu, 2015). Una CE mayor a $5 \mathrm{dS} \mathrm{m}^{-1}$ en la rizosfera, puede afectar negativamente la absorción de agua y nutrientes, lo que conlleva a disminuciones del rendimiento final (Bustomi et al., 2014; Santos y Torres, 2018).

En la preparación de las soluciones nutritivas hidropónicas se utilizan fertilizantes altamente solubles que generalmente tienen un costo elevado (Huang, 2009), mismo que se ha incrementado considerablemente en los últimos años. Aunado a ello, el agua para riego es también un recurso natural cada vez más limitado, por lo que su uso en sistemas hidropónicos debe ser muy eficiente (Salazar et al., 2014).

Esta solución nutritiva normalmente es aplicada mediante riego por goteo de alta frecuencia, permitiendo un drenaje o sobre-riego que ayude a mantener estable la concentración de nutrientes en la rizosfera (Sánchez et al., 2014). El sobre-riego de solución nutritiva que drena, normalmente ya no es utilizado por la planta, y puede perderse en el suelo o bien se puede recuperar mediante un sistema de recirculación para incorporarlo nuevamente al cultivo. Cuando la solución drenada 
no se reutiliza y se permite la infiltración en el sitio o se conduce fuera del invernadero, al sistema hidropónico se le conoce como abierto; por el contrario, si se recoge para volverse a usar en el cultivo, previa esterilización y ajuste de $\mathrm{pH}, \mathrm{CE}$ y concentración de nutrimentos, se le llama sistema cerrado (Alarcón, 2006).

Los sistemas abiertos, por su facilidad de manejo, representan la técnica hidropónica más ampliamente utilizada en México y en todo el mundo (Sandoval et al., 2012; Salazar et al., 2014), con la consecuente pérdidas de agua y fertilizantes que, en instalaciones grandes, pueden afectar negativamente al manto freático por la acumulación de altas cantidades de sales y con ello contaminar ríos, lagos y mares (Alarcón, 2006). Si la solución nutritiva que drena se reutiliza (sistemas hidropónicos cerrados), además de tener un ahorro económico de agua y fertilizantes, el daño ambiental es mucho menor (Nakano et al., 2010).

El problema principal con los sistemas cerrados es la dificultad técnica de su manejo ya que, con el paso del tiempo, puede ocurrir un desbalance de la solución nutritiva drenada debido a la acumulación de iones menos consumidos por la planta $\left(\mathrm{SO}_{4}{ }^{2-}, \mathrm{Ca}^{2+} \mathrm{y} \mathrm{Mg}^{2+}\right)$ más sales disueltas presentes en el agua de riego como $\mathrm{Na}^{+}$y $\mathrm{Cl}^{-}$, lo que al recircular va ocasionando un desequilibrio cada vez mayor de nutrientes y un incremento de la CE a niveles que afectan el crecimiento y rendimiento (Tunali et al., 2009; Van Os, 2009; Zhang et al., 2016).

Además, con los sistemas cerrados también existe un alto riesgo de dispersión de enfermedades sobre todo con los ciclos de cultivo tan largos como convencionalmente se maneja el jitomate (Sánchez et al., 2014). Entre más largo es el ciclo de cultivo, mayor es la posibilidad de que aparezcan enfermedades en la raíz y desequilibrios en la solución nutritiva, lo que eventualmente puede afectar el rendimiento respecto a sistemas sin recirculación; por ello, con frecuencia en la práctica comercial se reportan rendimientos menores en sistemas cerrados respectos a los abiertos en cultivos que se manejan en ciclos largos como el jitomate o chile pimiento, en los que durante varios meses coexisten etapas de crecimiento vegetativo con reproductivo (Savvas et al., 2009; Nakano et al., 2010).

Para que los sistemas hidropónicos cerrados se puedan implementar con mayor probabilidad de éxito y aprovechar sus ventajas, es conveniente buscar formas de manejo que sean sencillas para el productor, pero que no afecten negativamente el rendimiento o la calidad. Una estrategia que se podría probar para disminuir los riesgos de alteraciones en la $\mathrm{CE}$, desequilibrios nutricionales o dispersión de enfermedades con sistemas hidropónicos cerrados, sería producir jitomate con ciclos de cultivo muy cortos (menos de cuatro meses de trasplante a fin de cosecha) como el desarrollado en la Universidad Autónoma Chapingo como una alternativa al sistema convencional y que ha sido validado a escala comercial por varios productores (Sánchez et al., 2012).

Se piensa que este sistema podría adoptarse con ventajas para sistemas hidropónicos cerrados, pero hacen falta estudios concretos para su manejo particular. Por ello se llevó a cabo el presente estudio con el objetivo de comparar diferentes métodos de recirculación de la solución nutritiva contra un sistema abierto, en el comportamiento agronómico de un sistema de cultivo de jitomate manejado a tres racimos por planta para acortar a menos de cuatro meses el periodo de trasplante a fin de cosecha. De manera particular, se comparó el rendimiento que se obtuvo con dichos métodos y se determinó el ahorro en agua y nutrientes que se puede lograr con cada método de recirculación de la solución nutritiva respecto al sistema abierto (sin recirculación). 


\section{Materiales y métodos}

El experimento se llevó a cabo de julio a diciembre del año 2018 en un invernadero de $1000 \mathrm{~m}^{2}$ del Posgrado de Horticultura de la Universidad Autónoma Chapingo (UACH), en Texcoco, Estado de México, localizado a $19^{\circ} 29^{\prime} 35^{\prime \prime}$ de latitud norte y $98^{\circ} 52^{\prime} 19^{\prime \prime}$ de longitud oeste y una altura de 2250 m. Se utilizó el híbrido de jitomate Bullseye ${ }^{\circledR}$ de la Compañía Seminis, el cual es de tipo saladette y hábito de crecimiento semideterminado.

Las semillas fueron sembradas en una mezcla de sustrato (turba, perlita y arena de tezontle rojo a una proporción de 25,25 y 50\%, respectivamente) contenida en charolas de poliestireno de 60 cavidades con $250 \mathrm{~cm}^{3}$ por cavidad y una separación de $5 \mathrm{~cm}$ entre centro y centro de cavidades.

A partir de cinco días después de la siembra (dds) y hasta el trasplante (43 dds) se aplicaron en promedio dos riegos diarios con solución nutritiva que contenía las siguientes concentraciones de nutrimentos $\left(\mathrm{mg} \mathrm{L}^{-1}\right)$ : nitrógeno $(\mathrm{N})=100$, fósforo $(\mathrm{P})=25$, potasio $(\mathrm{K})=125$, calcio $(\mathrm{Ca})=125$, magnesio $(\mathrm{Mg})=20$, azufre $(\mathrm{S})=75$, fierro $(\mathrm{Fe})=2$, manganeso $(\mathrm{Mn})=1$, boro $(\mathrm{B})=0.5$, cobre $(\mathrm{Cu})=0.2$ y zinc $(\mathrm{Zn})=0.2$, que resultó en una $\mathrm{CE}$ de $1.5 \mathrm{dS} \mathrm{m}^{-1}$; el pH se ajustó a 6.5.

El trasplante se llevó a cabo en camas de cultivo rellenas de tezontle rojo de 1-3 mm de diámetro, de $1 \mathrm{~m}$ de ancho por $20 \mathrm{~m}$ de largo y $0.25 \mathrm{~m}$ de profundidad, con costados de tabique y pasillos cementados de $50 \mathrm{~cm}$ de ancho entre camas, forradas en el fondo con polietileno negro calibre 600, a lo largo de cada cama fue colocada una tubería de PVC de 2" de diámetro, ranurada en su base inferior a cada $50 \mathrm{~cm}$ para permitir la conducción de la solución nutritiva drenada hacia el extremo y ser colectada.

La disposición de las plantas fue de tres hileras por cama, a una separación de $35 \mathrm{~cm}$ entre hileras y $25 \mathrm{~cm}$ entre plantas, lo que dio una densidad de población de 12 plantas $\mathrm{m}^{-2}$ útil ( 8 plantas $\mathrm{m}^{-2} \mathrm{de}$ invernadero). Durante los primeros 15 días después del trasplante se aplicó la misma solución nutritiva que en el semillero para todos los tratamientos. De ahí en adelante la concentración de nutrientes en la solución nutritiva aplicada fue el doble (solución nutritiva al 100\%). Los tratamientos evaluados fueron los siguientes.

\section{Tratamiento 1}

Recirculación sin análisis químico de la solución drenante. En un tinaco de 5000 litros de capacidad, se preparaba la solución nutritiva al $100 \%$ de concentración. Se aplicaba en riego por goteo procurando un $20 \%$ de drenaje en cada riego. Cada vez que el tinaco se vaciaba, se preparaban $4000 \mathrm{~L}$ con una solución nutritiva nueva al 100\% de concentración más 1000 L de la solución drenada, colectada y desinfectada, sin que se realizaran correcciones de $\mathrm{pH}, \mathrm{CE}$ o de alguno de sus nutrientes.

\section{Tratamiento 2}

Con análisis químico de la solución drenada. En un tinaco de 5000 litros de capacidad, partiendo de una solución nutritiva al 100\% de concentración, se aplicaba en riego por goteo procurando un $20 \%$ de drenaje en cada riego. Cada vez que el tinaco se vaciaba, se preparaban $4000 \mathrm{~L}$ con una 
solución nutritiva ajustada selectivamente por elemento nutritivo ( $\mathrm{N}, \mathrm{P}, \mathrm{K}, \mathrm{Ca}, \mathrm{Mg}$ y $\mathrm{S}$ ) con base en un análisis químico del drenaje que se hacía cada 15 días, los 1000 L restantes fueron aportados con la solución nutritiva drenada, colectada y desinfectada.

\section{Tratamiento 3}

Con ajuste de la solución según absorción teórica. En un tinaco de 5000 L, partiendo de una solución nutritiva al $100 \%$ de concentración, se aplicaba en riego por goteo procurando un $20 \%$ de drenaje en cada riego. Cada vez que el tinaco se vaciaba, se preparaban 4000 L con una solución nutritiva ajustada selectivamente considerando una estimación de las tasas de absorción de las plantas de jitomate para cada elemento nutritivo (90\% para $\mathrm{N}, \mathrm{P}$ y K y $50 \%$ para $\mathrm{Ca}, \mathrm{Mg}$ y $\mathrm{S}$ ), respecto a la solución nutritiva inicial (Adams, 2004; Velazco et al., 2012; Urrestarazu, 2015), los $1000 \mathrm{~L}$ faltantes fueron complementados con la solución drenada, colectada y desinfectada.

\section{Tratamiento 4}

Testigo, con sistema abierto. En un tinaco de 5000 L de capacidad, se preparaba la solución nutritiva al $100 \%$ de concentración y se aplicaba en riego por goteo, procurando $20 \%$ de drenaje y cada vez que el tinaco se vaciaba, se preparaba nuevamente $100 \%$ del volumen inicial $(5000 \mathrm{~L})$ con la solución nutritiva completa.

La frecuencia de los riegos y la cantidad de solución nutritiva a aplicar diariamente procurando $20 \%$ de drenaje de solución nutritiva en cada tratamiento, se logró al aplicar entre 3 y 6 riegos diarios dependiendo de las condiciones climáticas y etapa fenológica del cultivo.

El drenaje de cada tratamiento fue colectado y almacenado temporalmente en tinacos de $1000 \mathrm{~L}$ antes de recircularlo, devolviéndolo cada quinto a séptimo día al tinaco de $5000 \mathrm{~L}$ que le correspondía según el tratamiento. Para desinfectar la solución drenada, previo a la recirculación se aplicó agua oxigenada (peróxido de hidrógeno al 50\%) a razón de $50 \mathrm{ml}$ de producto comercial por cada tinaco de $1000 \mathrm{~L}$ (60 ppm de $\mathrm{H}_{2} \mathrm{O}_{2}$ en solución).

Para el análisis químico de la solución drenada requerido para definir la concentración de sales en la preparación de la solución nutritiva en el tratamiento 2, se tomó del tinaco de 1000 L colectado de ese tratamiento una muestra de $500 \mathrm{ml}$, colocándola en frascos color ámbar para su análisis en el laboratorio. Para determinar el nitrógeno se utilizó el método de microkjeldahl, para fósforo se empleó el método de vanadato-molibdato, leyendo en un espectrofotómetro GENESYS 10 UV con una absorbancia de $420 \mathrm{~nm}$ y el potasio se determinó por medio de un flamómetro JENWAY (Chapman y Pratt, 1973).

Para medir calcio y magnesio se utilizó un espectrofotómetro de absorción atómica Pye Unicam SP 9 de Phillips y finalmente el azufre fue determinado por el método turbidimétrico con cloruro de bario (Chesnin y Yien, 1951). Las plantas fueron despuntadas (eliminación de la yema terminal del tallo principal) a los $81 \mathrm{dds}$, dejando dos hojas por arriba de la tercera inflorescencia formada. Todos los brotes laterales fueron eliminados conforme aparecieron, conduciendo el cultivo a un solo tallo por planta, de donde se cosecharon los frutos de los tres racimos. 
Se empleó un diseño de bloques completos al azar con cuatro tratamientos y siete repeticiones. La unidad experimental fue de $20 \mathrm{~m}^{2}$ (240 plantas). Los datos obtenidos fueron sometidos a análisis de varianza y comparaciones de medias con la prueba de Tukey $(p=0.05)$, usando el programa estadístico SAS (2002) versión 9.0. Se evaluaron variables morfológicas (altura de planta, diámetro de tallo y área foliar), de peso seco (total de planta y de fruto), y del rendimiento y sus componentes (peso medio y número de frutos).

La altura de planta $(\mathrm{cm})$, se midió de la base del tallo a la altura donde se hizo la poda del ápice. El diámetro de tallo $(\mathrm{mm})$ se midió con un vernier electrónico 'digimatic caliper' (Mitutoyo, modelo núm. CD-6 CS) a la altura de entre la primera y segunda inflorescencia. El área foliar por planta $\left(\mathrm{cm}^{2}\right)$ se determinó con el apoyo de un integrador de área foliar marca LI- 3000A, Lincoln, Nebraska y con este dato se estimó el índice de área foliar $\left(\mathrm{m}^{2}\right.$ hoja $\mathrm{m}^{-2}$ de área cubierta).

El peso seco total por planta (g) se midió con el apoyo de una balanza granataria y de estufa para su secado a $60{ }^{\circ} \mathrm{C}$ hasta peso constante. El peso seco de fruto (g) se midió con una balanza granataria secándolo al mismo tiempo que el resto de la planta, con este dato y con el de peso seco total por planta se estimó el índice de cosecha (peso seco de fruto/peso seco total de planta). El número de frutos (frutos $\mathrm{m}^{-2}$ ) se obtuvo de la suma de los frutos de calidad comercial obtenidos en cada corte.

El rendimiento total se calculó por unidad de superficie $\left(\mathrm{kg} \mathrm{m}^{-2}\right)$, sumando el peso de los frutos en cada uno de los cortes cosechados en cada $\mathrm{m}^{2}$. El peso medio de fruto $(\mathrm{g})$, se calculó dividiendo el rendimiento por unidad de superficie $\left(\mathrm{g} \mathrm{m}^{-2}\right)$ entre el número de frutos totales cosechados. Las variables morfológicas fueron medidas a los 102 dds y las de peso seco a los 143 dds. Además, se calculó el gasto de agua y de macronutrimentos y se hizo análisis de tejido vegetal de las plantas manejadas en cada uno de los tratamientos probados.

El gasto de agua $\left(\mathrm{L} \mathrm{m}^{-2}\right)$ se determinó sumando la cantidad de litros de solución nutritiva aplicados durante el periodo de trasplante a fin de cosecha, y el gasto de macronutrimentos $\left(\mathrm{g} \mathrm{m}^{-2}\right)$ se calculó con base en la concentración $\left(\mathrm{mg} \mathrm{L}^{-1}\right)$ de los nutrientes con la que se preparó la solución nutritiva en cada tratamiento y la cantidad (litros) aplicada a lo largo del ciclo de cultivo desde trasplante hasta fin de cosecha.

Dado que estas determinaciones se hicieron una vez que el ciclo de cultivo había finalizado, las comparaciones entre los distintos tratamientos, solamente se hicieron con base en los valores absolutos calculados, sin que se aplicaran pruebas estadísticas. El análisis de tejido vegetal también se realizó a los 143 dds, para lo cual se tomaron tres plantas por unidad experimental en cada una de las repeticiones. Con las tres plantas se hizo una muestra compuesta, la cual se secó en una estufa a $70{ }^{\circ} \mathrm{C}$.

Con un molino WILEY modelo 4 de 110 VAC, se molieron las muestras y luego fueron almacenadas en frascos de vidrio transparentes para su posterior digestión. En un matraz Kjeldahl se colocó $0.5 \mathrm{~g}$ de muestra molida, a la que se le agregaron $4 \mathrm{ml}$ de una mezcla de ácido sulfúrico y acido perclórico en proporción 4:1 más $1 \mathrm{ml}$ de agua oxigenada al 30\%, dando un extracto de color negro. Luego se colocó el matraz sobre una plancha caliente hasta que el extracto hirvió y cambió a un color claro. 
Después se dejó enfriar por una hora, entonces se procedió a aforar a $50 \mathrm{ml}$ con agua destilada. A partir de la solución obtenida con este procedimiento, se hicieron las determinaciones de nitrógeno, fósforo, potasio, calcio y magnesio (Alcántar y Sandoval, 1999). Para azufre, se llevó a cabo una digestión alterna similar, pero en este caso en la mezcla se utilizó ácido nítrico en vez de ácido sulfúrico en proporción 2:1 (Alcántar y Sandoval, 1999). La determinación de cada uno de los macronutrimentos se hizo con las mismas metodologías utilizadas para la solución nutritiva drenada arriba señaladas.

\section{Resultados y discusión}

\section{Variables morfológicas}

El análisis de varianza de este grupo de variables (datos no mostrados) indicó que hubo diferencia altamente significativa para altura de planta y significativa para diámetro de tallo, pero no para índice de área foliar. Las comparaciones de medias (Cuadro 1) muestran que, la altura de planta en el tratamiento con $20 \%$ de recirculación sin análisis químico de la solución drenada (T1), fue mayor respecto a los otros dos tratamientos de recirculación (T2 y T3) y que el testigo (T4).

Cuadro 1. Comparaciones de medias de variables morfológicas a los 102 días después de la siembra en jitomate cv. Bullseye con distintos métodos de recirculación de la solución nutritiva.

\begin{tabular}{cccc}
\hline $\begin{array}{c}\text { Tratamiento de } \\
\text { recirculación }\end{array}$ & $\begin{array}{c}\text { Altura de } \\
\text { planta }(\mathrm{cm})\end{array}$ & $\begin{array}{c}\text { Diámetro de } \\
\text { tallo }(\mathrm{mm})\end{array}$ & $\begin{array}{c}\text { Índice de área foliar }\left(\mathrm{m}^{2} \text { de hoja } \mathrm{m}^{-2}\right. \\
\text { de área cubierta })\end{array}$ \\
\hline $\begin{array}{c}\mathrm{T} 1 \text { (sin análisis) } \\
\text { T2 (con análisis) }\end{array}$ & $114.9 \mathrm{a}$ & $12.2 \mathrm{ab}$ & $5.15 \mathrm{a}$ \\
$\mathrm{T} 3$ (según absorción & $104.1 \mathrm{~b}$ & $13.8 \mathrm{a}$ & $5.33 \mathrm{a}$ \\
$\begin{array}{c}\text { teórica) } \\
\text { T4 (testigo, sin } \\
\text { recirculación) }\end{array}$ & $106 \mathrm{~b}$ & $12.9 \mathrm{ab}$ & $4.99 \mathrm{a}$ \\
DMS & $98.3 \mathrm{~b}$ & $11.4 \mathrm{~b}$ & $4.56 \mathrm{a}$ \\
\hline
\end{tabular}

Medias con la misma letra en cada columna son estadísticamente iguales (Tukey, $p=0.05$ ). DMS= diferencia mínima significativa.

No hay una explicación especial para el mayor crecimiento en altura de las plantas del tratamiento de recirculación simple de los drenajes (T1), posiblemente se explique por la influencia de diferencias en factores microclimáticos que se presentaron en las plantas desde el semillero y que afectaron diferencialmente su crecimiento en altura. Para diámetro de tallo, el tratamiento de recirculación con previo análisis de los drenajes (T2), superó al testigo sin recirculación, sin diferencias entre los otros tratamientos. En índice de área foliar (IAF), todos los tratamientos fueron estadísticamente iguales, con una oscilación entre 4.5 y 5.3.

Estos valores posiblemente sean altos para una óptima fotosíntesis neta, ya que de acuerdo con Sánchez y Moreno (2017), los valores óptimos de IAF para una mayor acumulación de materia seca diaria en jitomate manejado en invernadero y alta densidad de población deben ser entre $3 \mathrm{y}$ 4. Valores mayores a 5, provocan sombreado excesivo entre las plantas, aspecto que pudo notarse en el cultivo, lo que afecta la velocidad de fotosíntesis neta por planta y en consecuencia el rendimiento final (Taiz et al., 2014). 


\section{Variables de peso seco, índice de cosecha y rendimiento y sus componentes}

Tanto el análisis de varianza (datos no mostrados) como el de comparaciones de medias (Cuadro

2) indican que, para las variables peso seco total de planta, índice de cosecha, número de frutos por planta, peso medio de fruto y rendimiento, no hubo diferencias significativas entre tratamientos.

Cuadro 2. Comparaciones de medias de variables del rendimiento en jitomate $c v$ Bullseye con distintos métodos de recirculación de la solución nutritiva.

\begin{tabular}{cccccc}
\hline Tratamiento de recirculación & $\begin{array}{c}\text { Peso seco } \\
\text { total } \\
\left(\mathrm{g} \mathrm{planta}^{-1}\right)\end{array}$ & $\begin{array}{c}\text { Número } \\
\left(\text { frutos } \mathrm{m}^{-2}\right)\end{array}$ & $\begin{array}{c}\text { Peso } \\
\text { medio de } \\
\text { fruto }(\mathrm{g})\end{array}$ & $\begin{array}{c}\text { Rendimiento } \\
\left(\mathrm{kg} \mathrm{m}^{-2}\right)\end{array}$ & $\begin{array}{c}\text { Índice de cosecha } \\
\text { (ps de fruto/ps } \\
\text { total })\end{array}$ \\
\hline T1 (sin análisis) & $140.4 \mathrm{a}$ & $100.5 \mathrm{a}$ & $110.4 \mathrm{a}$ & $11.13 \mathrm{a}$ & $0.47 \mathrm{a}$ \\
T2 (con análisis) & $132 \mathrm{a}$ & $107.9 \mathrm{a}$ & $118.1 \mathrm{a}$ & $12.71 \mathrm{a}$ & $0.45 \mathrm{a}$ \\
T3 (según absorción teórica) & $118.4 \mathrm{a}$ & $104.6 \mathrm{a}$ & $110.7 \mathrm{a}$ & $11.6 \mathrm{a}$ & $0.48 \mathrm{a}$ \\
$\begin{array}{c}\text { T4 (testigo, sin } \\
\text { recirculación) }\end{array}$ & $131 \mathrm{a}$ & $109.3 \mathrm{a}$ & $108.6 \mathrm{a}$ & $11.79 \mathrm{a}$ & $0.44 \mathrm{a}$ \\
DMS & 45.7 & 13.3 & 10.41 & 1.58 & 0.1 \\
\hline
\end{tabular}

Medias con la misma letra en cada columna son estadísticamente iguales (Tukey, $p=0.05$ ). DMS= diferencia mínima significativa. Ps= peso seco.

Los resultados de peso seco e índice de cosecha obtenidos permiten suponer que, en jitomate manejado en un ciclo corto como el establecido en el presente estudio, con cualquiera de los sistemas de recirculación probados no se afecta el crecimiento de las plantas ni la distribución de la materia seca que se dirige hacia los frutos, respecto a un sistema abierto, resultados que coinciden con Sánchez et al. (2014), quienes, al evaluar diferentes sistemas de recirculación de la solución nutritiva en jitomate, también manejado en un ciclo corto, no encontraron diferencia alguna entre tratamientos para este tipo de variables.

En cambio, Tunali et al. (2009); Massa et al. (2010). Reportaron disminuciones en el crecimiento de las plantas de jitomate al utilizar un sistema de recirculación de la solución nutritiva, posiblemente porque condujeron el cultivo con el manejo convencional de ciclo largo (Sánchez et al., 2012), en donde con el paso del tiempo, se dan desequilibrios importantes en la solución nutritiva drenada (Tunali et al., 2009; Van Os, 2009).

El rendimiento osciló entre 11.13 y $12.71 \mathrm{~kg} \mathrm{~m}^{-2}$ de invernadero, en un período de 110 días de trasplante a fin de cosecha, lo que da la oportunidad de obtener tres ciclos de cultivo por año. Poniendo como ejemplo el promedio de rendimiento de los tres sistemas con recirculación (11.81 $\mathrm{kg} \mathrm{m}^{-2}$ ), se tendría un potencial de rendimiento anual de al menos $350 \mathrm{t} \mathrm{ha}^{-1}$, que es similar o incluso ligeramente mayor al reportado en las empresas con un alto desempeño técnico bajo el sistema de ciclo largo (Castellanos y Borbón, 2009).

Seguramente el período tan corto con que se logró finalizar la cosecha (menos de cuatro meses a partir del trasplante) permitió escapar los desequilibrios nutrimentales en la solución nutritiva recirculante que ocurren con el paso del tiempo (Tunali et al., 2009; Van Os, 2009; Sánchez et al., 2014), de manera que no hubo diferencias en rendimiento entre los sistemas cerrados y el sistema abierto estudiados. En otros trabajos de recirculación de la solución nutritiva con jitomate, en donde el ciclo de cultivo es mucho más largo, se han reportado disminuciones significativas en el rendimiento con respecto a los sistemas abiertos (Tunali et al., 2009; Van Os, 2009). 
Además, con el manejo del cultivo con ciclos largos, la recirculación promueve la aparición de enfermedades que poco a poco se diseminan en todo el cultivo afectando el rendimiento y calidad (Sánchez et al., 2014). En investigaciones similares con el sistema de alta densidad de población y despunte al tercer racimo se han logrado rendimiento de alrededor de $15 \mathrm{~kg} \mathrm{~m}^{-2}$ en un ciclo de cuatro meses de trasplante a fin de cosecha (Sánchez et al., 2012; Sánchez et al., 2014).

Sin embargo, el rendimiento obtenido en este estudio fue relativamente bajo, posiblemente por el alto índice de área foliar formado (alrededor de 5), lo que provocó sombreado entre las plantas y una mayor tasa de respiración de mantenimiento, afectándose la fotosíntesis neta diaria y con ello el rendimiento (Sánchez y Moreno, 2017).

De cualquier manera, con los resultados obtenidos se puede señalar que, cualquiera de los tratamientos de recirculación de la solución nutritiva evaluados, es susceptible de ser implementado en el sistema de producción de jitomate en ciclos cortos, sin menoscabo del rendimiento, con ventajas en ahorro de agua y fertilizantes respecto al tratamiento testigo sin recirculación como se muestra más adelante, y que además se evitaría la contaminación del manto freático como lo mencionan Nakano et al. (2010).

\section{Gasto de agua y fertilizantes}

En el Cuadro 3 se presentan las cantidades de agua y nutrimentos aportados por cada $\mathrm{m}^{2} \mathrm{de}$ invernadero durante el período de trasplante a fin de cosecha. Se observa que el gasto de agua fue de $437.6 \mathrm{~L} \mathrm{~m}^{-2}$ de invernadero para cada uno de los tratamientos con recirculación de solución nutritiva, mientras que para el tratamiento testigo sin recirculación (T4), el gasto fue de 547.2 L.

Cuadro 3. Gasto de agua ( $L$ ) y cantidad (g) de macronutrientes aplicados por $\mathbf{m}^{2}$ de invernadero en jitomate cv. Bullseye durante 110 días de trasplante a fin de cosecha con distintos métodos de recirculación de la solución nutritiva.

\begin{tabular}{cccccccc}
\hline Tratamiento de recirculación & Agua & Nitrógeno & Fósforo & Potasio & Calcio & Magnesio & Azufre \\
\hline T1 (sin análisis) & 437.6 & 91.3 & 22.4 & 116 & 120.8 & 20.8 & 68 \\
T2 (con análisis) & 437.6 & 97.6 & 26.4 & 132.8 & 116 & 16 & 66.4 \\
T3 (según absorción teórica) & 437.6 & 100 & 24.8 & 127.2 & 88 & 16 & 48 \\
T4 (testigo, sin recirculación) & 547.6 & 108.8 & 27.2 & 138.4 & 145.8 & 25.6 & 81.6 \\
\hline
\end{tabular}

Esta diferencia de $109 \mathrm{~L} \mathrm{~m}^{-2}$ se debió a que, en los primeros tres tratamientos, se recirculó la solución nutritiva drenada, correspondiente aproximadamente a un $20 \%$ del total aplicado. Extrapolando estos datos a una hectárea de invernadero y con el establecimiento de tres ciclos de cultivo al año, que es posible con el sistema de tres racimos por planta manejado, se obtendría un ahorro de agua aproximado de $3300 \mathrm{~m}^{3}$ al año por el hecho de recircular la solución nutritiva drenada, lo que representa una disminución considerable en el uso de este recurso.

Benoit y Ceustermans (1995) citados por Gul (2011) mencionan que, para cultivos sin suelo, con 15 a $25 \%$ de sobre-riego de solución nutritiva, se pierden aproximadamente $2900 \mathrm{~m}^{3} \mathrm{ha}^{-1}$ año $^{-1}$, similar a lo observado en este estudio. Por su parte, Sánchez et al. (2014) reportaron ahorros de $32.6 \%$ en agua al comparar un sistema con recirculación de solución nutritiva respecto a uno sin recirculación en el cultivo de jitomate cultivado en camas rellenas con sustrato de tezontle. 
Cabe resaltar que el ahorro de agua con la recirculación de la solución nutritiva resulta de gran importancia agronómica, en particular para su implementación en zonas en donde el agua es un recurso cada vez más escaso para la producción de cultivos. En el Cuadro 3 también se observó que, con base en los valores absolutos calculados, en los tres métodos de recirculación de la solución nutritiva, el aporte de nutrimentos fue menor respecto al testigo.

Con el tratamiento con recirculación de la solución nutritiva sin análisis (T1) se aplicó menos nitrógeno, fósforo y potasio (17.5, 4.8 y $22.4 \mathrm{~g} \mathrm{~m}^{-2}$ de invernadero menos que el testigo, esto es aproximadamente un $16 \%$ para cada nutrimento), mientras que con el tratamiento de recirculación según la absorción teórica establecida (T3), se aportó menos calcio, magnesio y azufre (57, 9.6 y $33.6 \mathrm{~g} \mathrm{~m}^{-2}$ de invernadero menos que el testigo, correspondiente a 39,37 y $41 \%$ para cada nutrimento, respectivamente). En el tratamiento T1, se esperaría un ahorro de $20 \%$ de todos los nutrimentos respecto al testigo; sin embargo, el ahorro fue de $16 \%$ debido a que en este tratamiento se continuó aplicando la solución nutritiva completa hasta un mes después del trasplante.

En la preparación de la solución nutritiva en el tratamiento T3 para $\mathrm{Ca}, \mathrm{Mg}$ y S se suministró solamente $50 \%$ de lo aplicado en la solución completa; sin embargo, el ahorro no fue de esa magnitud en virtud de que la recirculación de la solución nutritiva en este tratamiento también se inició un mes después del trasplante. Al respecto Sánchez et al. (2014) en un experimento con recirculación de solución nutritiva en camas de tezontle en el cultivo de jitomate, exhibieron aportes de macronutrientes de 59.3, 31, 135 y $93.3 \mathrm{~g} \mathrm{~m}^{-2}$ para nitrógeno, fósforo, potasio y calcio, respectivamente.

Al extrapolarse los datos obtenidos a una ha de invernadero y tres ciclos de cultivo al año, el ahorro con el tratamiento T1 respecto al testigo sería del orden de $525 \mathrm{~kg}$ de nitrógeno, $144 \mathrm{~kg}$ de fósforo y $672 \mathrm{~kg}$ de potasio, mientras que con el tratamiento T3 el ahorro respecto al testigo sería de 1734 $\mathrm{kg}$ de calcio, $288 \mathrm{~kg}$ de magnesio y $1008 \mathrm{~kg}$ de azufre, que desde un punto de vista económico resulta muy importante para el productor.

\section{Análisis nutrimental}

El análisis de varianza (datos no mostrados) y de comparaciones de medias (Cuadro 4) muestra que entre tratamientos no hubo diferencias significativas para ninguno de los nutrientes determinados en tejido vegetal, lo que sugiere que las plantas absorbieron nutrimentos en proporciones similares independientemente de la concentración a que se haya preparado la solución nutritiva. Con estos resultados también se puede señalar que con el manejo de jitomate en ciclos cortos se pueden lograr ahorros considerables en fertilizantes con cualquiera de los métodos de recirculación probados, respecto al testigo, sin que se afecte la tasa de absorción de nutrientes por las plantas (Adams, 2004).

De manera general, también se observa (Cuadro 4) que las plantas absorbieron una mayor cantidad de potasio, seguido de nitrógeno y calcio, y en menor proporción fósforo, magnesio y azufre, resultados que concuerdan con Quesada y Bertsch (2013); Vargas-Canales (2014) en estudios similares con jitomate. Cabe señalar, que los porcentajes de macronutrientes absorbidos por las plantas estuvieron dentro de los rangos de suficiencia reportados por Sánchez (2004) para el cultivo de jitomate, indicando que, con cualquiera de los sistemas de manejo evaluados, las plantas tuvieron disponibles los elementos necesarios para su crecimiento y desarrollo normal (Sánchez, 2004; Alcántar et al., 2016). 
Cuadro 4. Comparaciones de medias del contenido de macronutrientes expresado en g y en (\%) en plantas de jitomate $c v$ Bullseye a los 101 días después del trasplante, con distintos métodos de recirculación de solución nutritiva.

\begin{tabular}{|c|c|c|c|c|c|c|}
\hline $\begin{array}{l}\text { Tratamiento de } \\
\text { recirculación }\end{array}$ & Nitrógeno & Fósforo & Potasio & Calcio & Magnesio & Azufre \\
\hline T1 (sin análisis) & $3.47 \mathrm{a}(2.45)$ & $0.93 a(0.66)$ & $6.38 \mathrm{a}(4.6)$ & $3.2 \mathrm{a}(2.27)$ & $1.14 \mathrm{a}(0.8)$ & $0.94 \mathrm{a}(0.65)$ \\
\hline T2 (con análisis) & $3.09 \mathrm{a}(2.32)$ & $0.87 \mathrm{a}(0.66)$ & 5.94a (4.5) & 2.91a (2.2) & $0.66 \mathrm{a}(0.5)$ & $0.68 \mathrm{a}(0.51)$ \\
\hline $\begin{array}{l}\text { T3 (absorción } \\
\text { teórica) }\end{array}$ & $2.74 \mathrm{a}(2.3)$ & $0.7 \mathrm{a}(0.59)$ & $5.13 \mathrm{a}(4.17)$ & $2.29 \mathrm{a}(1.95)$ & $0.61 \mathrm{a}(0.52)$ & $0.64 a(0.54)$ \\
\hline $\begin{array}{c}\text { T4 (testigo, } \\
\text { sistema abierto) }\end{array}$ & $2.77 \mathrm{a}(2.12)$ & $0.92 \mathrm{a}(0.71)$ & $5.58 \mathrm{a}(4.3)$ & $3 a(2.27)$ & $0.65 a(0.5)$ & $0.46 a(0.35)$ \\
\hline DMS & 1.74 & 0.3 & 1.6 & 1.43 & 0.58 & 0.54 \\
\hline
\end{tabular}

Medias con la misma letra en cada columna son estadísticamente iguales (Tukey, $p=0.05$ ). DMS= diferencia mínima significativa.

\section{Conclusiones}

Con el manejo de las plantas de jitomate en alta densidad de población y despunte para cosechar solamente tres racimos, en un ciclo de producción tan corto como 110 días de trasplante a fin de cosecha, se hace factible recircular un $20 \%$ de la solución nutritiva con o sin un análisis de la misma o bien considerando una tasas de absorción de las plantas de $90 \%$ para N, P y K y de $50 \%$ para Ca, $\mathrm{Mg}$ y S, respecto a la solución nutritiva inicial, sin que se presenten desbalances nutricionales, ni proliferación de enfermedades de la raíz, de manera que, al comparar estos métodos con un sistema abierto, no se afecta el crecimiento ni el rendimiento de las plantas, ahorrándose agua y fertilizantes y haciendo más sostenible el proceso de producción.

\section{Literatura citada}

Adams, P. 2004. Aspectos de la nutrición mineral en cultivos sin suelo en relación al suelo. In: tratado de cultivo sin suelo. (Ed.). Urrestarazu, G. M. Ediciones Mundi-Prensa. Madrid, España. 81-111 pp.

Alarcón, V. A. 2006. Proyectos en cultivo sin suelo ¿Cómo empezar? In: cultivos sin suelo. Alarcón, V. A. (Ed.). Compendios de horticultura 17. Ediciones de Horticultura, SL. Reus. España. 11-21 pp.

Alcántar, G. G. y Sandoval, V. M. 1999. Manual de análisis químico de tejido vegetal guía de muestreo, preparación, análisis e interpretación. Sociedad Mexicana de la Ciencia del Suelo, AC. Chapingo, Estado de México. Publicación Especial 10. 156 p.

Alcántar, G. G.; Trejo-Téllez, L. I.; Fernández, P. L. y Rodríguez, M. M. N. 2016. Elementos esenciales. In: nutrición de cultivos. Alcántar, G. G.; Trejo-Téllez, L. I. y Gómez, M. F. C. (Ed). Editorial BBA. Texcoco, Estado de México. 21-55 pp.

Bustomi, R. R. A.; Masateru, S.; Diding, S. and Ahmad, T. 2014. The effect of EC levels of nutrient solution on the growth, yield, and quality of tomatoes (Solanum lycopersicum) under the hydroponic system. J. Agric. Eng. and Biotech. 2(1):7-12.

Chapman, H. D. y Pratt, P. E. 1973. Métodos de análisis de suelos, plantas y agua. Traducido al español por Contin, A. (Ed.). Trillas. México, DF. 195 p. 
Chesnin, L. and Yien, C. H. 1951. Turbidimetric determination of available sulphur. Proceedings of Soil Science Society of America. 149-151 pp.

Gul, A.; Tuzel, Y.; Tuzel, I. H.; Irget, M. E.; Kidoglu, F. and Tepecik, M. 2011. Effects of nutrition and irrigation on sweet pepper production in volcanic tuff. Span. J. Agric. Res. 9(1):221-229.

Huang, W. Y. 2009. Factors contributing to the recent increase in U.S. fertilizer prices, 2002-08. Agricultural resources situation and outlook number AR-33. U.S. Department of Agriculture, Economic Research Service, Washington, DC. 21 p.

Liang, W.; Jiang, Y. and Zhang, Y. 2006. Accumulation of soil soluble salt in vegetable greenhouses under heavy application of fertilizers. Agric. J. 1(3):123-127.

Massa, D.; Incrocci, L.; Maggini, R.; Carmassi, G.; Campiotti, C. A. and Pardossi, A. 2010. Strategies to decrease water drainage and nitrate emission from solilless culture of greenhouse tomato. Agric. Water Manag. 97(7):971-980.

Nakano, Y.; Sasaki, H.; Nakano, A.; Suzuki, K. and Takaichi, M. 2010. Growth and yield of tomato plants as influenced by nutrient application rates with quantitative control in closed rockwool cultivation. J. Japan. Soc. Hort. Sc. 79(1):47-55.

Quesada-Roldán, G. y Bertsch-Hernández, F. 2013. Obtención de la curva de extracción nutrimental del híbrido de tomate FB-17. Terra Latinoam. 31(1)1-7.

Raviv, M. and Lieth, H. 2008. Significance of soilless culture in agriculture. In: soilless culture. Theory and practice. Raviv, M. and Lieth, H. (Eds.). Elsevier. Amsterdam, the Netherlands. 1-11 pp.

Salazar, M. R.; Rojano, A. A. y López, C. I. L. 2014. La eficiencia en el uso del agua en la agricultura controlada. Tecnología y Ciencias del Agua. 5(2):177-183.

Sánchez, C. F. y Moreno, P. E. C. 2017. Diseño agronómico y manejo de invernaderos. Universidad Autónoma Chapingo (UACH). Chapingo, Texcoco, Estado de México. 405 p.

Sánchez, C. F.; Moreno, P. E. C.; Pineda, P. J.; Osuna, J. M.; Rodríguez, P. J. E. y Osuna, E. T. 2014. Producción hidropónica de jitomate (Solanum lycopersicum L.) con y sin recirculación de la solución nutritiva. Agrociencia. 48(2):185-197.

Sánchez, P. A. 2004. Análisis y diagnóstico nutricional en los cultivos sin suelo. In: tratado de cultivo sin suelo. Urrestarazu, G. M. (Ed). $3^{\text {a }}$. Ediciones Mundi-Prensa, Madrid, España. 49-79 pp.

Sánchez-Del, C. F.; Moreno, P. E. C. and Contreras, M. E. 2012. Development of alternative comercial soilless production systems I. Tomato. Acta Hortic. 947:179-187.

Sandoval, V. M., Sánchez, G. P. y Alcántar, G. G. 2012. Principios de la hidroponía y del fertirriego. In: nutrición de cultivos. Alcántar, G. G. y Trejo-Téllez, L. I. (Ed.). Colegio de Postgraduados. Campus Montecillo, Texcoco, Estado de México. 373-438 pp.

Santos, B. M. and Torres-Quezada, E. A. 2018. Irrigation and fertilization. In: tomatoes. $2^{\text {nd }}$ Edition, (Ed.). Heuvelink, E. CAB International, Wallingford, UK. 180-206 pp.

SAS. Institute. 2002. Statistical Analysis System. SAS/STAT 9.1 user's guide. Cary, N. C. USA.

Savvas, D.; Sigrimis, N.; Chatzieustratiou, E. and Paschalidis, C. 2009. Impact of a progressive $\mathrm{Na}$ and $\mathrm{Cl}$ accumulation in the root zone on pepper grown in a closed-cycle hydroponic system. Acta Hort. 807:451-456.

SIAP. 2018. Servicio de Información Agroalimentaria y Pesquera. Atlas Agroalimentario. SAGARPA. Ciudad de México, México.

Sonneveled, C. and Voogt, W. 2009. Substrates: chemical characteristics and preparation. In: plant nutrition of greenhouse crops. Sonneveld, C. and Vogt, W. (Eds.). Springer. 227-252 pp. 
Taiz, L.; Zeiger, E.; Ian, M. and Morphy, A. 2014. Plant physiology and development. 6 (Ed.). Sinauer associates, Inc. Publisher. Suderland, Massachusetts, USA. 761 p.

Takahashi, K. 1984. Injury by continuous cropping in vegetables: various problems in the cultivation using grafted plants. Yasaishikenjo Kenkyu Shiryo. 18:87-89.

Tunali, U.; Tüzel, I. H.; Tüzel, Y. and Öztekin, G. B. 2009. Effects of salinity on tomato in a closed system. Acta Hort. 807:457-462.

Urrestarazu, G. M. 2015. Manual práctico del cultivo sin suelo e hidroponía. (Ed.). Mundi-Prensa. Madrid, España. 267 p.

Van-Os, E. 2009. Comparison of some chemical and non-chemical treatments to disinfect a recirculating nutrient solution. Acta Hort. 843:229-234.

Vargas-Canales, J. M.; Castillo-González, A. M.; Pineda-Pineda, J.; Ramírez-Arias, J. A. y AvitiaGarcía, E. 2014. Extracción nutrimental de jitomate (Solanum lycopersicum L.) en mezclas de tezontle con aserrín nuevo y reciclado. Rev. Chapingo Ser. Hortic. 20(1):71-88.

Velasco, H. E.; Nieto, Á. R. y Navarro, L. E. R. 2012. Cultivo del tomate en hidroponía e invernadero. Tercera edición, Primera reimpresión. Universidad Autónoma Chapingo (UACH). Chapingo, Estado de México. 126 p.

Zhang, P.; Senge, M. and Dai, Y. 2016. Effects of salinity stress on growth, yield, fruit quality and water use efficiency of tomato under hydroponics system. Reviews in Agricultural Science. $4: 46-55$. 